University of Wollongong

Research Online

Faculty of Engineering and Information

Faculty of Engineering and Information

Sciences - Papers: Part B

Sciences

2019

\title{
An innovative gynecological HDR brachytherapy applicator system for treatment delivery and real-time verification
}

Anna Romanyukha

University of Wollongong, ar947@uowmail.edu.au

Mauro Carrara

Fondazione Irccs Istituto Nazionale Tumori

Davide Mazzeo

Universita degli Studi di Milano

Chiara Tenconi

Universita degli Studi di Milano

Tebarak Al-Salmani

University of Wollongong

See next page for additional authors

Follow this and additional works at: https://ro.uow.edu.au/eispapers1

Part of the Engineering Commons, and the Science and Technology Studies Commons

Research Online is the open access institutional repository for the University of Wollongong. For further information contact the UOW Library: research-pubs@uow.edu.au 


\title{
An innovative gynecological HDR brachytherapy applicator system for treatment delivery and real-time verification
}

\author{
Abstract \\ The multichannel vaginal cylinder (MVC) applicator employed for gynecological high dose rate (HDR) \\ brachytherapy increases dose delivery complexity, and thus makes the treatment more prone to errors. A \\ quality assurance (QA) procedure tracking the source throughout dose delivery can detect dwell position \\ and time errors in the multiple channels of the applicator. \\ A new MVC system with integrated real time in vivo treatment delivery QA has been developed based on \\ diodes embedded on the outer surface of the MVC. It has been pre-calibrated and verified using a non- \\ clinical treatment plan with consecutive test positions and dwell times within each catheter, followed by \\ the delivery of ten clinical plans of adjuvant vaginal cuff brachytherapy following hysterectomy for \\ endometrial cancer.
}

The non-clinical verification showed overall mean dwell position and time discrepancies between the nominal and measured treatment of $-0.2 \pm 0.5 \mathrm{~mm}$ and $-0.1 \pm 0.1 \mathrm{~s}(\mathrm{k}=1)$, respectively. The clinical plans showed mean positional discrepancies of $0.2 \pm 0.4$ and $0.0 \pm 0.8 \mathrm{~mm}$, for the central and peripheral catheters, respectively, and mean dwell time discrepancies of $-0.1 \pm 0.2$ and $-0.0 \pm 0.1 \mathrm{~s}$ for central and peripheral catheters, respectively.

The innovative prototype of the MVC system has shown the ability to track the source with sub-mm and sub-second accuracy, and demonstrated potential for its incorporation into the clinical routine.

\section{Disciplines}

Engineering | Science and Technology Studies

\section{Publication Details}

Romanyukha, A., Carrara, M., Mazzeo, D., Tenconi, C., Al-Salmani, T., Poder, J., Cutajar, D., Fuduli, I., Petasecca, M., Bucci, J., Cerrotta, A., Pappalardi, B., Piccolo, F., Pignoli, E. \& Rosenfeld, A. (2019). An innovative gynecological HDR brachytherapy applicator system for treatment delivery and real-time verification. Physica Medica: an international journal devoted to the applications of physics to medicine and biology, 59 151-157.

\section{Authors}

Anna Romanyukha, Mauro Carrara, Davide Mazzeo, Chiara Tenconi, Tebarak Al-Salmani, Joel Poder, Dean L. Cutajar, lolanda Fuduli, Marco Petasecca, Joseph A. Bucci, Annamaria Cerrotta, Brigida Pappalardi, Federica Piccolo, Emanuele Pignoli, and Anatoly B. Rosenfeld 


\title{
An innovative gynecological HDR brachytherapy applicator system for treatment delivery and real-time verification
}

\author{
Anna Romanyukha ${ }^{1}$, Mauro Carrara ${ }^{2, *}$, Davide Mazzeo ${ }^{3}$, Chiara Tenconi ${ }^{4}$, Tebarak Al-Salmani ${ }^{1}$, \\ Joel Poder ${ }^{5}$, Dean Cutajar ${ }^{1}$, Iolanda Fuduli ${ }^{1}$, Marco Petasecca ${ }^{1}$, Joseph Bucci ${ }^{5}$, Annamaria \\ Cerrotta $^{6}$, Brigida Pappalardi ${ }^{6}$, Federica Piccolo $^{6}$, Emanuele Pignoli $^{2}$, Anatoly Rosenfeld $^{1}$ \\ ${ }^{1}$ Centre for Medical Radiation Physics, University of Wollongong, Wollongong, NSW, Australia \\ ${ }^{2}$ Medical Physics Unit, Fondazione IRCCS Istituto Nazionale Tumori, Milan, Italy \\ ${ }^{3}$ Department of Physics, Università degli Studi di Milano, Milan, Italy \\ ${ }^{4}$ Department of Oncology and Hemato-Oncology, Università degli Studi di Milano, Milan, Italy \\ ${ }^{5}$ St George Cancer Care Center, Kogarah, NSW, Australia \\ ${ }^{6}$ Radiation Oncology 2 Unit, Fondazione IRCCS Istituto Nazionale Tumori, Milan, Italy
}

Keywords: HDR brachytherapy; multichannel vaginal applicator; source tracking; diodes; in vivo treatment QA

\author{
*Corresponding author: \\ Mauro Carrara \\ Medical Physics Unit, Dept. of Diagnostic Imaging and Radiotherapy \\ Fondazione IRCCS Istituto Nazionale dei Tumori \\ Via Venezian, 1 \\ 20133 Milano \\ Phone: +390223902123 \\ Fax: +390223902124 \\ e-mail: mauro.carrara@istitutotumori.mi.it
}




\begin{abstract}
The multichannel vaginal cylinder (MVC) applicator employed for gynecological high dose rate (HDR) brachytherapy increases dose delivery complexity, and thus makes the treatment more prone to errors. A quality assurance (QA) procedure tracking the source throughout dose delivery can detect dwell position and time errors in the multiple channels of the applicator.

A new MVC system with integrated real time in vivo treatment delivery QA has been developed based on diodes embedded on the outer surface of the MVC. It has been pre-calibrated and verified using a non-clinical treatment plan with consecutive test positions and dwell times within each catheter, followed by the delivery of ten clinical plans of adjuvant vaginal cuff brachytherapy following hysterectomy for endometrial cancer.

The non-clinical verification showed overall mean dwell position and time discrepancies between the nominal and measured treatment of $-0.2 \pm 0.5 \mathrm{~mm}$ and $-0.1 \pm 0.1 \mathrm{~s}(\mathrm{k}=1)$, respectively. The clinical plans showed mean positional discrepancies of $0.2 \pm 0.4$ and $0.0 \pm 0.8 \mathrm{~mm}$, for the central and peripheral catheters, respectively, and mean dwell time discrepancies of $-0.1 \pm 0.2$ and $-0.0 \pm$ $0.1 \mathrm{~s}$ for central and peripheral catheters, respectively.

The innovative prototype of the MVC system has shown the ability to track the source with sub$\mathrm{mm}$ and sub-second accuracy, and demonstrated potential for its incorporation into the clinical routine.
\end{abstract}




\section{Introduction}

The multichannel vaginal cylinder (MVC) applicator has been widely adopted in gynecological high dose rate (HDR) brachytherapy (BT) treatments. It has shown reductions in doses received by organs-at-risk, as compared to the single channel vaginal cylinder (SVC) applicator, without compromising the dose to the target. In fact, as compared to SVC the MVC geometry provides more degrees of freedom (i.e., the source is also dwelling in peripheral channels) to optimize the dose distribution for each patient [1-5].

The presence of multiple channels increases treatment complexity, and thus makes HDR treatments more prone to error. Errors can arise as a result of transfer tube misconnections, afterloader malfunction, and mistakes in afterloader calibration, among others, and thus affect source stepping distances, dwell times, and dwell positions within the applicator. Moreover, treatment errors often remain unknown due to a lack of real-time or post-treatment dose delivery verification in the current treatment flow $[6,7]$.

Treatment verification is recommended in order to ensure optimal outcomes for the patient, and can be done by measuring or visualizing the HDR source during dose delivery either as a quality assurance (QA) procedure performed prior to treatment delivery, or for in vivo treatment QA. Methods have been investigated with systems such as ion chamber [8] and two-dimensional (2D) diode arrays [9], pixelated detectors [10], Gallium Nitride (GaN) probes [11], electronic portal imaging devices (EPIDs) [12], plastic scintillators [13,14], diamond detectors [15], fluorescent screen imaging [16], thermoluminescence dosimeters (TLDs) [17] and Alanine [18]. However, to the best of our knowledge, among the listed methods only GaN-based dosimeters have been used to perform in vivo QA of treatments delivered with MVCs. Alternative source tracking systems that allow sub-millimeter and sub-second dwell position and dwell time verification in real-time should therefore still be investigated. 
The aim of this study was to produce and assess an innovative prototype of a MVC applicator with embedded and pre-calibrated diode detectors that can provide in vivo and real time QA of the delivered treatment by comparing it to the prescribed plan.

\section{Materials and Methods}

\subsection{MVC prototype description}

The applicator prototype was designed and manufactured at the Centre for Medical Radiation Physics (CMRP) at the University of Wollongong. It is $30 \mathrm{~mm}$ in diameter and contains seven equally spaced peripheral channels and one central cavity to host the peripheral and central catheters of the commercially available Vaginal CT/MR Multi Channel Applicator (Elekta Brachytherapy, TH Veenendaal, NL), respectively. In particular, the peripheral catheters are disposable plastic catheters with a diameter of $2 \mathrm{~mm}$ and an external length of $293 \mathrm{~mm}$ (i.e., "catheter with collar 6F") and the central catheter is the reusable Intravaginal CT/MR Tube. Epitaxial (epi) diode detectors were selected for HDR source tracking due to their high sensitivity and radiation hardness, and embedded on the applicator surface. The diodes are fabricated on ptype epitaxial silicon with a sensitive volume of $1.5 \times 1.5 \times 0.5 \mathrm{~mm}^{3}$. They are connected to an inhouse developed reader based on the multichannel electrometer Application Specific Integrated Circuit (ASIC) that is able to accommodate up to 32 detectors for simultaneous readout. Even though the current integration of the diode is performed at an interval of $1 \mathrm{~ms}$, the output signal was averaged to 50 measurements to increase the signal to noise ratio, resulting in a final readout frequency of $20 \mathrm{~Hz}$. Accompanying readout software was also developed at the CMRP to display the instantaneous and integral responses of the connected detectors in real-time. The raw output signal of each detector is automatically stored for post-measurement analysis. A more detailed description and characterization of the epi-diodes and data acquisition (DAQ) readout system is provided elsewhere $[9,19]$. 
The applicator prototype was assembled by placing three diodes into dedicated grooves with an angular interval of about 120 degrees around the surface of the MVC applicator at distances of 35,37 , and $39 \mathrm{~mm}$ from the applicator tip, respectively, ensuring a detection coverage of the proximal 6-7 $\mathrm{cm}$ of the vagina. Since in adjuvant vaginal cuff BT following hysterectomy, as a routine, it is recommended that the proximal $3-5 \mathrm{~cm}$ of the vagina be treated [20], the selected diode arrangement would be appropriate to cover the clinically significant region. A phantom of $160 \times 220 \times 160 \mathrm{~mm}^{3}$ was fabricated using polymethylmethacrylate (PMMA) to surround the applicator without any air gaps and ensure adequate scattering conditions. A picture of the experimental setup used to perform all measurements of this study is shown in figure 1 . The MVC system is positioned inside the PMMA phantom with transfer tube connections in all existing channels. The three diodes are connected to the DAQ system for readout, and read out in real time throughout the irradiation outside the BT bunker.

\subsection{Afterloader description}

Measurements were performed using the Flexitron afterloading unit (Elekta Brachytherapy, TH Veenendaal, NL) at the BT facility of the Fondazione IRCSS Istituto Nazionale dei Tumori (INT) in Milan, Italy. The afterloader contains an Ir-192 source with a $3.5 \mathrm{~mm}$ active length (Flexisource, Elekta) that is attached to a steel wire [21] and can be delivered using a total of 40 available treatment channels. The Air Kerma Strength $S_{K}$ of the source ranges between about 45 (when it is installed in the afterloader) and $20 \mathrm{mGy} \mathrm{m}^{2} \mathrm{~h}^{-1}$ (when it is removed and replaced). The unit allows a minimal step size and dwell time of $1 \mathrm{~mm}$ and 0.1 seconds, respectively. The positional accuracy of the system is $\pm 0.5 \mathrm{~mm}[22]$.

\subsection{MVC system calibration}

The first part of the study concerns the calibration of the MVC system - that is to assign to the measured response of the diodes a source dwell time and position (with respect to a specific 
reference point) within the applicator catheters of the MVC system. The dwell position was defined as the center of the active length of the source. The origin of the reference system was set on the upper surface of the MVC applicator and the absolute location of the first dwell position in each of the seven peripheral applicator channels was determined using GafChromic film measurements. The film was wrapped around the applicator surface, aligning the edge with the tip of the applicator, and the source was sent to the first dwell position (i.e., the most distal with respect to the afterloader) for a dwell time of 3 seconds. Position locations exposed on the GafChromic film were evaluated using a Matlab (The Mathworks, Natick, MA, USA) script to determine the center of each Ir-192 exposure and its location with respect to the edge of the film. Measurements were repeated three times, and the obtained average locations of the first dwell position, given as the distance $(\mathrm{mm})$ from the applicator surface, $\pm 0.1 \mathrm{~mm}(\mathrm{k}=1)$, were $7.1 \mathrm{~mm}$, $6.9 \mathrm{~mm}, 6.8 \mathrm{~mm}, 7.1 \mathrm{~mm}, 6.9 \mathrm{~mm}, 6.5 \mathrm{~mm}$ and $6.5 \mathrm{~mm}$, for the peripheral catheters 1 to 7 , respectively. The first dwell position in the central catheter was set as $5.5 \mathrm{~mm}$ and was taken from the Elekta user manual.

\subsubsection{MVC calibration for source dwell position detection}

To calibrate the MVC system in terms of source position detection, the relationship between diode response and source position was investigated. The source was sent to dwell positions of $1100-1288 \mathrm{~mm}$ in the peripheral channels and $1100-1300 \mathrm{~mm}$ in the central channel, respectively, at a step size of $1 \mathrm{~mm}$ and a dwell time of 1 second in each position. $1288 \mathrm{~mm}$ and $1300 \mathrm{~mm}$ are the first dwell positions (i.e., maximal distal positions) that can be set for the peripheral and central catheters, respectively.

The output was normalized by $\mathrm{S}_{\mathrm{K}}$ at the time of measurement and averaged over all the readout values corresponding to a near-constant diode response, and thus indicating a single programmed dwell position (i.e., readouts corresponding to the source transiting from one dwell position to the other when the response of the diode was monotonically variable were eliminated). The obtained 
mean responses $\overline{\mathrm{R}}_{\text {diode }}$ were plotted as a function of the source distance $\mathrm{d}_{\text {cath }}$ from the applicator tip, and those belonging to the two diodes with the higher signals were fitted with multi-term Gaussian functions according to equation 1:

$$
\overline{\mathrm{R}}_{\text {diode }}\left(d_{\text {cath }}\right)=\mathrm{a}_{1} \cdot \mathrm{e}^{\left(\frac{d_{c a t h}-\mathrm{b}_{1}}{\mathrm{c}_{1}}\right)^{2}}+\mathrm{a}_{2} \cdot \mathrm{e}^{\left(\frac{d_{c a t h}-\mathrm{b}_{2}}{\mathrm{c}_{2}}\right)^{2}}+\cdots \quad \text { Eq. } 1
$$

where $a_{n}, b_{n}$ and $c_{n}$ are the fitting parameters of the $n$-th term of the equation.

Thus, once the multi-term Gaussian functions are defined for the source dwelling in every available catheter they can be used for finding the source dwelling position $d_{\text {cath }}$ in the given catheter at any time. The multi-term Gaussian-interpolated diode response function (normalized by the source $\mathrm{S}_{\mathrm{K}}$ ) provides two possible solutions for the source dwell position. The ambiguity of source position determination is avoided by taking into account the response of a second diode located in a slightly different depth relative to the tip of the catheter in comparison to the diode with highest response, as shown in figure 2 .

A schematic of the applicator with the three diodes positioned on its surface is shown in figure 2 . An example of the diode response with the source dwelling in the central catheter is plotted as a function of the source distance from the applicator tip. In this example, the diodes 1 and 3 were selected as reference diodes for dwell position and time reconstruction.

\subsubsection{MVC calibration for source dwell time detection}

Source dwell time is determined from the plateaus in the diode signal i.e., the constancy of diode response with respect to the time. Dwell time is calculated as the ratio of the number of measurements in each plateau and the frequency of readout, set at $20 \mathrm{~Hz}$ throughout this study. An example of a plot with clearly distinguishable consecutive dwelling position plateaus is given in figure 3 for dwells of five second duration. 
The system's ability to reconstruct Ir-192 dwell positions and dwell times using the established methodology was first verified using a test plan with multiple consecutive dwell positions in every one of the eight applicator catheters. Chosen dwell positions with a step-size of $10 \mathrm{~mm}$ covered a range of $60 \mathrm{~mm}$ and $70 \mathrm{~mm}$ (i.e., from 5.5 to $75.5 \mathrm{~mm}$ from the applicator tip) in the peripheral and central channels, respectively, and were delivered for a dwell time of 5 seconds in each position. The absolute differences $\Delta \mathrm{p}$ and $\Delta \mathrm{t}$ between nominal and measured dwell positions and times, respectively, were then computed for every source position according to $\Delta \mathrm{p}=\mathrm{p}_{\text {meas }}$ $\mathrm{p}_{\text {nom }}$ and $\Delta \mathrm{t}=\mathrm{t}_{\text {meas }}-\mathrm{t}_{\text {nom }}$.

Following the first verification study, a total of ten BT treatments previously delivered at the Fondazione IRCCS Istituto Nazionale dei Tumori to cervical cancer patients employing the 30mm MVC, were randomly selected from the database and delivered in-phantom. Overall, 181 and $106 \mathrm{dwell}$ positions for the source dwelling in the central and in the lateral catheters, respectively, were planned. Discrepancies between reconstructed and nominal dwell positions and times were evaluated as described above.

\section{Results}

The quality of fitting parameters derived for the diode response functions used for dwell position reconstruction in each catheter can be seen in table 1 . The two diodes with the highest signal, the number of Gaussian terms used for the interpolation curve, and their corresponding $\mathrm{R}^{2}$ values are provided.

Following calibration of the MVC system, dwell position-time plots as those shown in figure 4 could be obtained for treatment delivery verification. An example of the source dwelling in the central catheter during the delivery of the non-clinical plan (a), and of a complete clinical treatment plan (i.e., central and the six peripheral channels $\mathrm{C} 2$ to $\mathrm{C} 7$ ) (b), are provided.

The distributions of all resulting dwell time and position discrepancies for the non-clinical plan (i.e., step-size of $10 \mathrm{~mm}$, dwell time $5 \mathrm{~s}$ in all available catheters) are given in figure 5, with the 
mean discrepancies for each single catheter reported in table 2. Overall mean dwell position and time discrepancies were determined as $-0.2 \pm 0.5 \mathrm{~mm}$ and $-0.1 \pm 0.1 \mathrm{~s}(\mathrm{k}=1)$, respectively. $50 \%$ and $95 \%$ of absolute positional discrepancies were within $0.4 \mathrm{~mm}$ and $1.3 \mathrm{~mm}$, respectively, whereas $50 \%$ and $95 \%$ of absolute time discrepancies were within $0.1 \mathrm{~s}$ and $0.4 \mathrm{~s}$, respectively.

Discrepancy distributions between nominal and reconstructed dwell positions and times for all 10 delivered clinical treatment plans are plotted in figure 5. The mean dwell position discrepancies are $0.2 \pm 0.4 \mathrm{~mm}$ and $0.0 \pm 0.8 \mathrm{~mm}(\mathrm{k}=1)$ for the central and lateral catheters, respectively. The mean dwell time discrepancies are $-0.1 \pm 0.2 \mathrm{~s}$ and $-0.0 \pm 0.1 \mathrm{~s}(\mathrm{k}=1)$ for the central and lateral catheters, respectively.

In general, $50 \%$ and $95 \%$ of absolute positional discrepancies $|\Delta \mathrm{p}|$ were $<0.3 \mathrm{~mm}$ and $<0.8 \mathrm{~mm}$ for the source dwelling in the central catheter, respectively, and $<0.5 \mathrm{~mm}$ and $<1.5 \mathrm{~mm}$ for the source dwelling in the peripheral catheters, respectively. Overall, 96.2 and $77.9 \%$ of absolute positional discrepancies were $<1 \mathrm{~mm}$ for the central and peripheral channels, respectively.

$50 \%$ and $95 \%$ of absolute dwell time discrepancies $|\Delta \mathrm{t}|$ were $<0.05 \mathrm{~s}$ and $<0.40 \mathrm{~s}$, respectively, for the source dwelling in the central catheter, and $<0.05 \mathrm{~s}$ and $<0.20 \mathrm{~s}$, respectively, for the source dwelling in the peripheral catheters.

Figure 6 provides a graphical visualization of the correspondence between planned (i.e., nominal) and measured dwell positions and times for the central and lateral catheters of the 10 delivered clinical treatment plans. Higher discrepancies between planned and real dwell positions and times are shown by the higher gaps between the red (nominal) and blue (measured) data points.

\section{Discussion}

In this study, an innovative prototype of a MVC applicator with embedded diode detectors was produced. Preliminary measurements on a non-clinical and on 10 different clinical treatment plans have demonstrated the MVC system's ability to monitor the HDR source throughout the entire dose delivery phase, providing in vivo and real time QA of the delivered treatment by 
comparing it to the prescribed plan. The system is in principle able to detect errors in dwell position and dwell times, including spontaneous afterloader malfunction, incorrect applicator and indexer lengths, mistakes in transfer tube connections, source calibration, and administration of the incorrect treatment plan.

A great advantage is that the MVC system can be pre-calibrated via a relatively easy method, and source positions and times can be obtained in real time without any assumptions or particular dose distribution calculations within the applicator/patient. In fact, source positions are constrained along the central and peripheral catheters, and the diodes have fixed positions with respect to the catheters. Thus, for each possible source position in one of the eight treatment channels, a unique solution exists from the combination of the two dosimeters with the highest responses. This solution can be determined experimentally prior to the use of the applicator and is valid without further recalibration in the case of stable diode response. Therefore, only a QA of the diode stability with time is required. Moreover, specific corrections for the possible energy and angular dependences of the diodes were not necessary, because the overall diode response was taken as reference and intrinsically modeled with the multi-term Gaussian functions.

This is different to systems that are not built-in with the applicator, such as $2 \mathrm{D}$ arrays $[8,9]$ or EPIDs [12], where more complex assumptions, pre-calibrations and calculations have to be performed to accurately reconstruct source positions inside the patient. For a correct calibration of the MVC system, it is however very important that it is calibrated in the same conditions that are present during treatment. Therefore, in order to ensure full-scattering conditions, a phantom was fabricated to surround the applicator during calibration, without leaving any air gaps at the applicator-phantom interface.

Another advantage of the proposed system over previously developed methods is that the diodes used for Ir-192 tracking are incorporated directly over the dose delivery instrument, i.e., MVC applicator, eliminating the introduction of any additional steps into the existing treatment procedure for the radiation oncologist or the radiation therapy technologist. Prior to patient 
irradiation the diode detectors must simply be connected to the readout system. For full system integration into the QA workflow, the readout system should ideally be connected with the afterloader treatment console for a direct comparison between nominal (i.e., planned on the console) and delivered (detected with the MVC system and calculated directly on the console) dwell position and times.

The proposed pre-calibrated MVC system is the first prototype of the multichannel vaginal cylindrical applicator with embedded diode detectors that can provide dwell positions and times with generally sub-mm and sub-second accuracy in real time. To the best of our knowledge, it is the first time ever that such a system is presented in literature. In fact, Wang et al [23] and Guiral et al [24] also equipped a commercial MVC applicator with four GaN-based dosimeters. They proposed a method for accurate in vivo determination of source dwell times and positions as a QA of the delivered treatment plan. Despite the very promising error detectability shown and evaluated in-phantom by means of testing intentionally introduced error protocols, a drawback of the proposed system remains in the reduction of peripheral channels available for treatment. The GaN-based dosimeters were hosted in four of the available treatment channels, thus significantly limiting the dose distribution optimization potential of the MVC. Moreover, in Guiral et al, the results mainly concern the use of the central catheter and only a simple test was performed on a three channel instrumented applicator.

If comparing the results limited to the central catheter, mean dwell position and time discrepancies are comparable. In particular, the discrepancies were $-0.11 \pm 0.7 \mathrm{~mm}$ and $0.2 \pm 0.4$ $\mathrm{mm}(\mathrm{k}=1)$ for dwell positions in Guiral et al and in our study, respectively, and $0.05 \pm 0.09 \mathrm{~s}$ and $-0.1 \pm 0.2 \mathrm{~s}(\mathrm{k}=1)$ for dwell times, respectively. In our study, it was additionally shown that comparable accuracies can also be obtained for lateral catheters on 10 clinical treatments, with resulting dwell position and time discrepancies of $0.0 \pm 0.8 \mathrm{~mm}$ and $-0.0 \pm 0.1 \mathrm{~s}(\mathrm{k}=1)$, respectively. 
This study clearly shows feasibility of the method and a potential for future use of the MVC system in the clinical routine. In principle, the diodes could be integrated to commercially available MVC applicators of any diameter, establishing the MVC system with applicators that are already clinically approved and familiar to operators. If these commercially available MVC systems will be designed with dedicated grooves that accommodate individual diodes and exactly fit the dimensions of the detectors, it will likely be possible that diodes could be removed and put back after each sterilization cycle without the need of a new calibration. On the contrary, recalibration will be necessary any time the diodes need to be replaced with new ones. Studies are still ongoing to define the most convenient adjustment to properly integrate the diodes with commercial applicators of different diameters

A range of source detection within the applicator of the proximal $60-70 \mathrm{~mm}$ of the vagina is enough for the majority of clinical applications of adjuvant vaginal cuff BT after hysterectomy, since the proximal $3-5 \mathrm{~cm}$ of the vagina are usually treated [20]. The current detection range limitation is determined by a combination of diode sensitivity and their location over the applicator. An increased number of diodes and their optimal distribution over the applicator could in principle allow an extension of this range. Time and spatial resolutions are also in principle good and would already allow the detection of significant delivery errors. A thorough evaluation of the system's ability to detect specific treatment errors will be conducted in the future.

Nevertheless, it must be noted that some improvements could still be accomplished to realize an advanced version of the MVC system. In particular, since the diodes are particularly small and since the reader is able to accommodate up to 32 detectors for simultaneous readout, additional diodes could be added over the MVC surface. There could in principle be one diode at every inter-channel space and some of them could also be placed closer to the applicator tip or to the applicator end. In this case more than two diode responses could be used to mathematically calculate the source position. Such an advanced diode assembly could possibly further improve source detection accuracy, as for instance: i) in the tails of the current calibration curves, where 
diode signal is lower (e.g. close to the tip of the applicator); ii) in the peak of the current calibration curves, where the source is dwelling directly above the sensitive volume of the diode and it is not possible to distinguish two consecutive dwell positions because these provide a similar response.

Even if, in the latter case, the second diode, located a few mm away, is able to accurately identify the correct position between the two, an exception occurred in our study for source positions 35.5 and $38.5 \mathrm{~mm}$ in catheter 2, where both of the diodes were not able to distinguish the two consecutive dwell positions, reconstructing both as $37.1 \mathrm{~mm}$. By eliminating the current physical restraint of the system, i.e., the presence of seven peripheral catheters and only three diodes, resulting in catheter 2 having two diodes of a similar response, spatial uncertainties in close proximity to the diode's sensitive volume could possibly be decreased.

An intrinsic limitation of the developed method is that the source tracking system is incorporated to the MVC applicator, but no conclusions can be drawn about the anatomical delivery of the delivered dose distribution. The use of the system will therefore provide a valuable validation that the delivered treatment is in accordance with the planned one, but no conclusions can be drawn about the delivered dose distribution. In vivo dose measurements in the urethra or rectum $[5,6,11,13,17,18,25]$ will additionally be needed to provide a comprehensive evaluation of the treatment. Further in vivo measurements are required to validate a possible combination of the dosimetry and source tracking methods.

\section{Conclusion}

The innovative prototype of a pre-calibrated MVC applicator with embedded diodes has shown the ability to track the HDR Ir-192 source during vaginal BT treatments with generally sub-mm and sub-second accuracy. The system is able to provide online verification of source dwell positions and times in real time for treatment verification, and there is potential for its future 
application in the clinical routine. Thorough studies to analyze the MVC system's ability to detect specific treatment errors and to investigate the possible combination of the MVC system with in vivo dosimetry methods will be conducted in the future.

\section{Acknowledgements}

We thank the Australian Research Council for scholarship support of A.A.R. through Laureate Fellowship grant FL130100116. This work was partially supported by the Italian Ministry of Health (5 x 1000 Funds - year 2014).

\section{References}

[1] Bahadur AY, Constantinescu C, Hassouna AH, Eltaher MM, Ghassal NM, NesreenAA. Single versus multichannel applicator in high-dose-rate vaginal brachytherapyoptimized by inverse treatment planning. J Contemp Brachytherapy. 2015;6:362-70.

[2] Carrara M, Romanyukha A, Tenconi C, Mazzeo D, Cerrotta A, Borroni M, et al. Clinical application of MOSkin dosimeters to rectal wall in vivo dosimetry in gy-necological HDR brachytherapy. Phys Med. 2017; 41:5-12.

[3] Carrara M, Cusumano D, Giandini T, Tenconi C, Mazzarella E, Grisotto S et al. Comparison of different treatment planning optimization methods for vaginal HDR brachytherapy with multichannel applicators: A reduction of the high doses to the vaginal mucosa is possible. Phys Med. 2017; 44:58-65.

[4] Demanes DJ, Rege S, Rodriguez RR, Schutz KL, Altieri GA, Wong T. The use and advantages of a multi-channel vaginal cylinder in high-dose-rate brachytherapy. Int J Radiat Oncol Biol Phys. 1999;44:211-9.

[5] Tanderup K, Lindegaard JC. Multi-channel intracavitary vaginal brachytherapy using threedimensional optimization of source geometry. Radiother Oncol. 2004;70:81-5. 
[6] Kertzscher G, Rosenfeld A, Beddar S, Tanderup K, Cygler JE. In vivo dosimetry: trends and prospects for brachytherapy. Br J Radiol. 2014;87(1041):20140206.

[7] Tanderup K, Beddar S, Andersen CE, Kertzscher G, Cygler JE. In vivo dosimetry in brachytherapy. Med Phys. 2013; 40(7).

[8] Manikandan A, Biplab S, David PA, Holla R, Vivek TR, Sujatha N. Relative dosimetrical verification in high dose rate brachytherapy using two-dimensional detector array IMatriXX. J Med Phys. 2011;36(3):171-5.

[9] Espinoza A, Petasecca M, Fuduli I, Howie A, Bucci J, Corde S, et al. The evaluation of a 2D diode array in "magic phantom" for use in high dose rate brachytherapy pretreatment quality assurance. Med Phys. 2015;42(2):663-73.

[10] Safavi-Naeini M, Han Z, Alnaghy S, Cutajar D, Petasecca M, Lerch ML, et al. BrachyView, a novel in-body imaging system for HDR prostate brachytherapy: Experimental evaluation. Med Phys. 2015;42(12):7098-107.

[11] Cartwright LE, Suchowerska N, Yin Y, Lambert J, Haque M, McKenzie DR. Dose mapping of the rectal wall during brachytherapy with an array of scintillation dosimeters. Med Phys. 2010;37(5):2247-55.

[12] Smith RL, Haworth A, Panettieri V, Millar JL, Franich RD. 3D catheter reconstruction in HDR prostate brachytherapy for pre-treatment verification using a flat panel detector. Phys Med. 2017; 39:121-131.

[13] Therriault-Proulx F, Briere TM, Mourtada F, Aubin S, Beddar S, Beaulieu L. A phantom study of an in vivo dosimetry system using plastic scintillation detectors for real-time verification of 192Ir HDR brachytherapy. Med Phys. 2011;38(5):2542-51.

[14] Johansen JG, Rylander S, Buus S, Bentzen L, Hokland SB, Søndergaard CS et al . Timeresolved in vivo dosimetry for source tracking in brachytherapy. Brachytherapy. $2018 ; 17: 122-132$ 
[15] Nakano T, Suchowerska N, McKenzie D, Bilek M. Real-time verification of HDR brachytherapy source location: implementation of detector redundancy. Phys Med Biol. 2005;50(2):319.

[16] Lightstone AW. Fluorescent screen for high-dose-rate (HDR) brachytherapy quality assurance. Med Dosim. 2005;30(3):143-4.

[17] Anton M, Wagner D, Selbach HJ, Hackel T, Hermann RM, Hess CF et al. In vivo dosimetry in the urethra using alanine/ESR during (192)Ir HDR brachytherapy of prostate cancer-a phantom study. Phys Med Biol. 2009; 54: 2915-31

[18] Jaselskè E, Adlienė D, Rudžianskas V, Urbonavičius BG, Inčiūra A. In vivo dose verification method in catheter based high dose rate brachytherapy. Phys Med. 2017; 44:1-10.

[19] Aldosari AH, Petasecca M, Espinoza A, Newall M, Fuduli I, Porumb C, et al. A two dimensional silicon detectors array for quality assurance in stereotactic radiotherapy: MagicPlate-512. Med Phys. 2014;41(9):091707.

[20] Small W, Beriwal S, Demanes DJ, Dusenbery KE, Eifel P, Erickson B, Jones E, Rownd JJ, De Los Santos JF, Viswanathan AN, Gaffney D; American Brachytherapy Society. American Brachytherapy Society consensus guidelines for adjuvant vaginal cuff brachytherapy after hysterectomy. Brachytherapy. 2012;11:58-67.

[21] Perez-Calatayud J, Ballester F, Das RK, DeWerd LA, Ibbott GS, Meigooni AS, et al. Dose calculation for photon-emitting brachytherapy sources with average energy higher than 50 keV: report of the AAPM and ESTRO. Med Phys. 2012;39(5):2904-29.

[22] Van Der Laarse R, Niatsetski Y. Flexitron and MicroSelectron-HDR. PDR Afterloaders' Transit Time and Dose (Elekta AB, Stockholm, Sweden, 2014). 2014.

[23] Wang R, Ribouton J, Pittet P, Guiral P, Jalade P, Lu G-N. Implementation of GaN based real-time source position monitoring in HDR brachytherapy. Rad Meas. 2014;71:293-6. 
[24] Guiral P, Ribouton J, Jalade P, Wang R, Galvan JM, Lu GN, et al. Design and testing of a phantom and instrumented gynecological applicator based on $\mathrm{GaN}$ dosimeter for use in high dose rate brachytherapy quality assurance. Med Phys. 2016;43(9):5240-51.

[25] Gambarini G, Carrara M, Tenconi C, Mantaut N, Borroni M, Cutajar D et al. Online in vivo dosimetry in high dose rate prostate brachytherapy with MOSkin detectors: in phantom feasibility study. Appl Radiat Isot. 2014; 83:222-226 


\section{Figure captions}

Figure 1: (a) Cylindrical multichannel applicator with the peripheral catheters and three diodes positioned around its surface (the central catheter is not shown in the image); (b) Two cylindrical components of the MVC, with the possibility of using the intrauterine tube in the innermost piece of the applicator, and the custom-made PMMA phantom to surround the applicator; (c) experimental setup used for the study.

Figure 2: Multi-term Gaussian functions in the central catheter where the cross-sectional horizontal view (a) of the applicator shows the Ir-192 source (red sphere) in the central channel, and the three color-coded diodes positioned on the applicator surface. Corresponding diode response curves are plotted as a function of the source distance from the applicator tip (b), and in this case the diodes with the highest response i.e., diodes 1 and 3 are used for position and dwell time reconstruction. The source in the dwell position is shown in the longitudinal view (c) with diodes 1 and 3 fixed around the applicator perimeter.

Figure 3: Example of normalized diode response as seen during treatment time. Points between the plateaus are due to the transit of the source from one dwell position to the next one.

Figure 4: Example of treatment delivery verification for a) catheter 1 of the test plan and for b) a complete clinical treatment plan (i.e., central and the six peripheral channels $\mathrm{C} 2$ to $\mathrm{C} 7$ ). The reconstructed and nominal dwell positions, shown in blue and red, respectively, are viewed as a function of treatment time. Plot $\mathrm{b}$ shows that the treatment starts in the furthest position from the applicator tip of the central catheter, delivering a total of 16 dwell positions of various dwell times. At 222.3 seconds the central channel delivery is completed (marked by the red arrow). The source then proceeds to two dwell positions $5 \mathrm{~mm}$ apart in peripheral channel 2 for 34.1 and 11.8 $\mathrm{s}$ in duration, respectively, and one dwell position in channel 3. The source then dwells in two 
positions in channel $4,1 \mathrm{~mm}$ apart and lasting $33.7 \mathrm{~s}$ and $11.3 \mathrm{~s}$, respectively, and then to one dwell position in consecutive channels 5, 6, and 7 for dwell times of $33.0 \mathrm{~s}, 21.7 \mathrm{~s}$, and $33.3 \mathrm{~s}$, respectively.

Figure 5: Discrepancy distributions between nominal and reconstructed dwell positions (a) and times (c) for the non-clinical treatment, respectively, and for the 10 delivered clinical treatment plans (i.e., (b) and (d), respectively). For the clinical treatment, distinct distributions for the central and the peripheral catheters are shown. Histograms are fitted with Gaussian distributions, which are also plotted with continuous lines.

Figure 6: Correspondence between the planned (red squares) and measured (blue dots) dwell positions (a) and times (c) for the central catheter, respectively, and between planned and measured dwell positions (b) and times (d) for the lateral catheters, respectively, in the 10 delivered clinical patient plans. 
Tables

Table 1. Calibration curve parameters for each catheter.

\begin{tabular}{|c|c|c|c|c|c|c|}
\hline \multirow{2}{*}{ Catheter } & \multicolumn{2}{|c|}{ Diodes } & \multicolumn{2}{c|}{ No. of Gaussian terms } & \multicolumn{2}{c|}{$\mathbf{R}^{2}$ values } \\
\cline { 2 - 7 } & HIGH & LOW & HIGH & LOW & HIGH & LOW \\
\hline C1 & D2 & D3 & 4 & 3 & 1 & $>0.99$ \\
\hline C2 & D2 & D3 & 4 & 3 & 1 & 1 \\
\hline C3 & D3 & D2 & 4 & 2 & 1 & $>0.99$ \\
\hline C4 & D3 & D1 & 4 & 2 & 1 & $>0.99$ \\
\hline C5 & D1 & D3 & 4 & 2 & $>0.99$ & $>0.99$ \\
\hline C6 & D1 & D2 & 3 & 2 & $>0.99$ & $>0.99$ \\
\hline C7 & D2 & D1 & 4 & 2 & 1 & $>0.99$ \\
\hline Central & D1 & D3 & 3 & 3 & 1 & 1 \\
\hline
\end{tabular}


Table 2: Results of the non-clinical dwell position and dwell time verification study with mean absolute differences $\Delta \mathrm{p}$ and $\Delta \mathrm{t}$ between the nominal and measured dwell positions and times for each catheter within the MVC, respectively.

\begin{tabular}{|c|c|c|}
\hline \multicolumn{3}{|c|}{ Catheter Discrepancies } \\
\hline CATHETER & $\Delta \mathbf{p ~ ( m m )}$ & $\Delta \mathbf{t}(\mathbf{s})$ \\
\hline C1 & $-0.2 \pm 0.3$ & $-0.2 \pm 0.1$ \\
\hline C2 & $0.0 \pm 0.4$ & $-0.2 \pm 0.1$ \\
\hline C3 & $-0.1 \pm 0.4$ & $-0.2 \pm 0.1$ \\
\hline C4 & $-0.5 \pm 0.6$ & $-0.1 \pm 0.2$ \\
\hline C5 & $-0.3 \pm 0.5$ & $-0.1 \pm 0.0$ \\
\hline C6 & $-0.1 \pm 0.5$ & $-0.2 \pm 0.2$ \\
\hline C7 & $-0.6 \pm 0.5$ & $-0.1 \pm 0.1$ \\
\hline Central & $-0.1 \pm 0.3$ & $-0.1 \pm 0.0$ \\
\hline
\end{tabular}


Figures
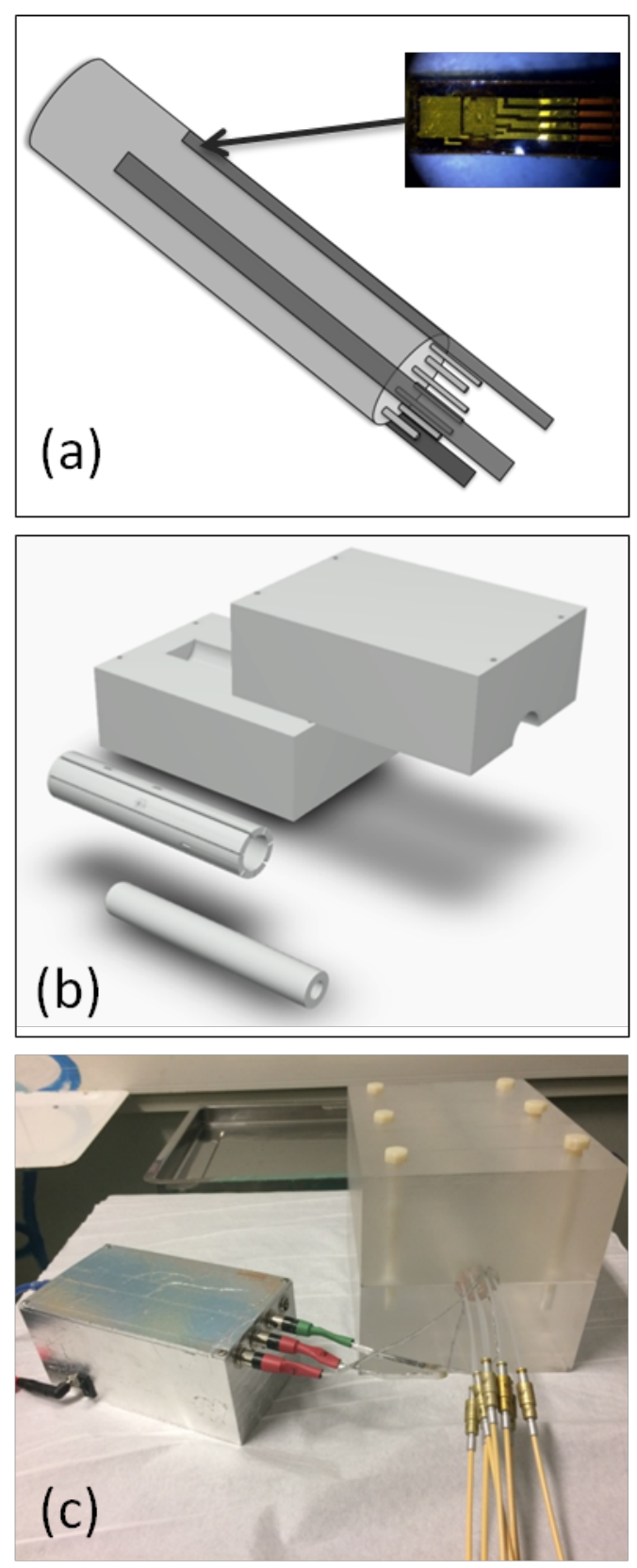

Figure 1 
(a)

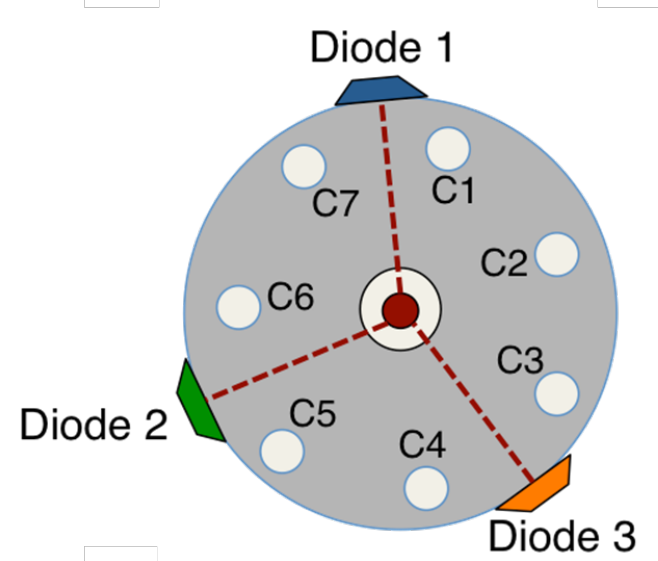

(c) (b)
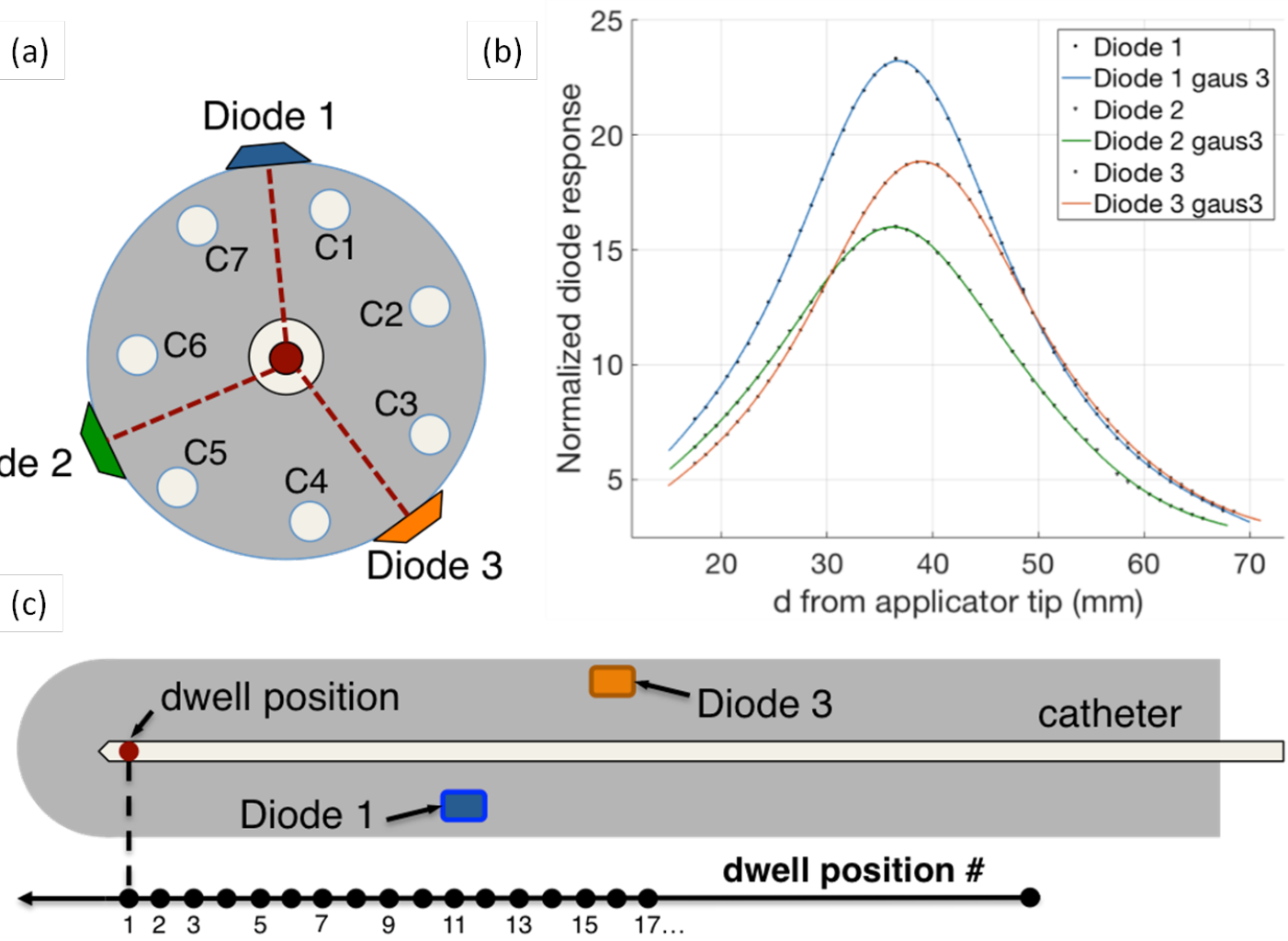

Figure 2 


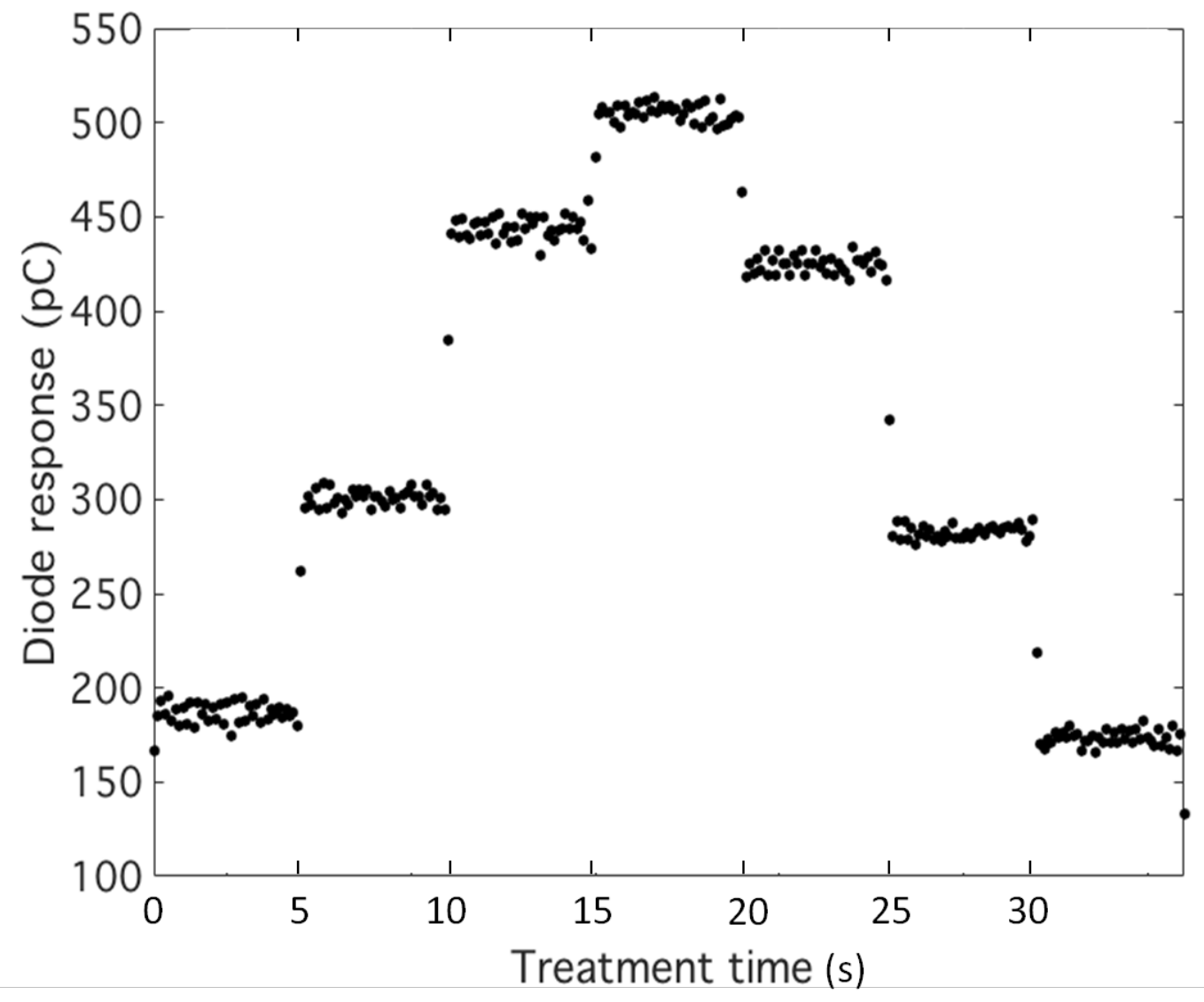

Figure 3 

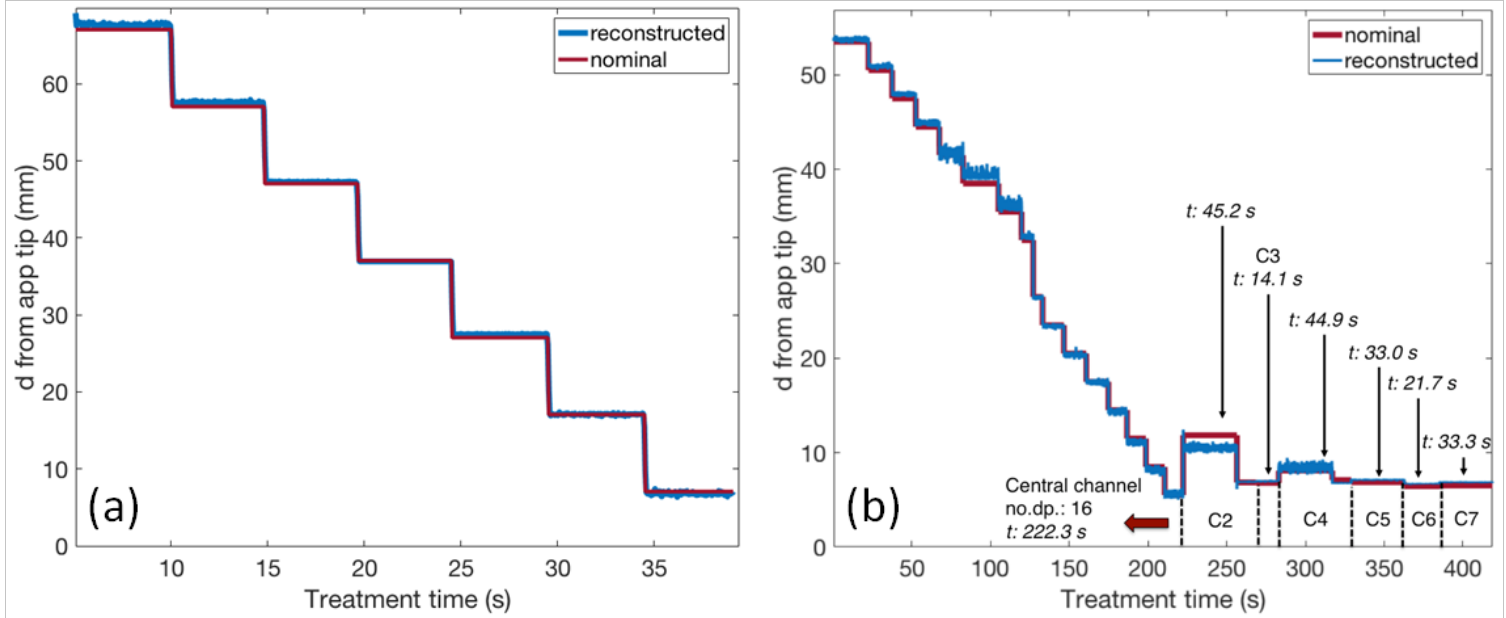

Figure 4 

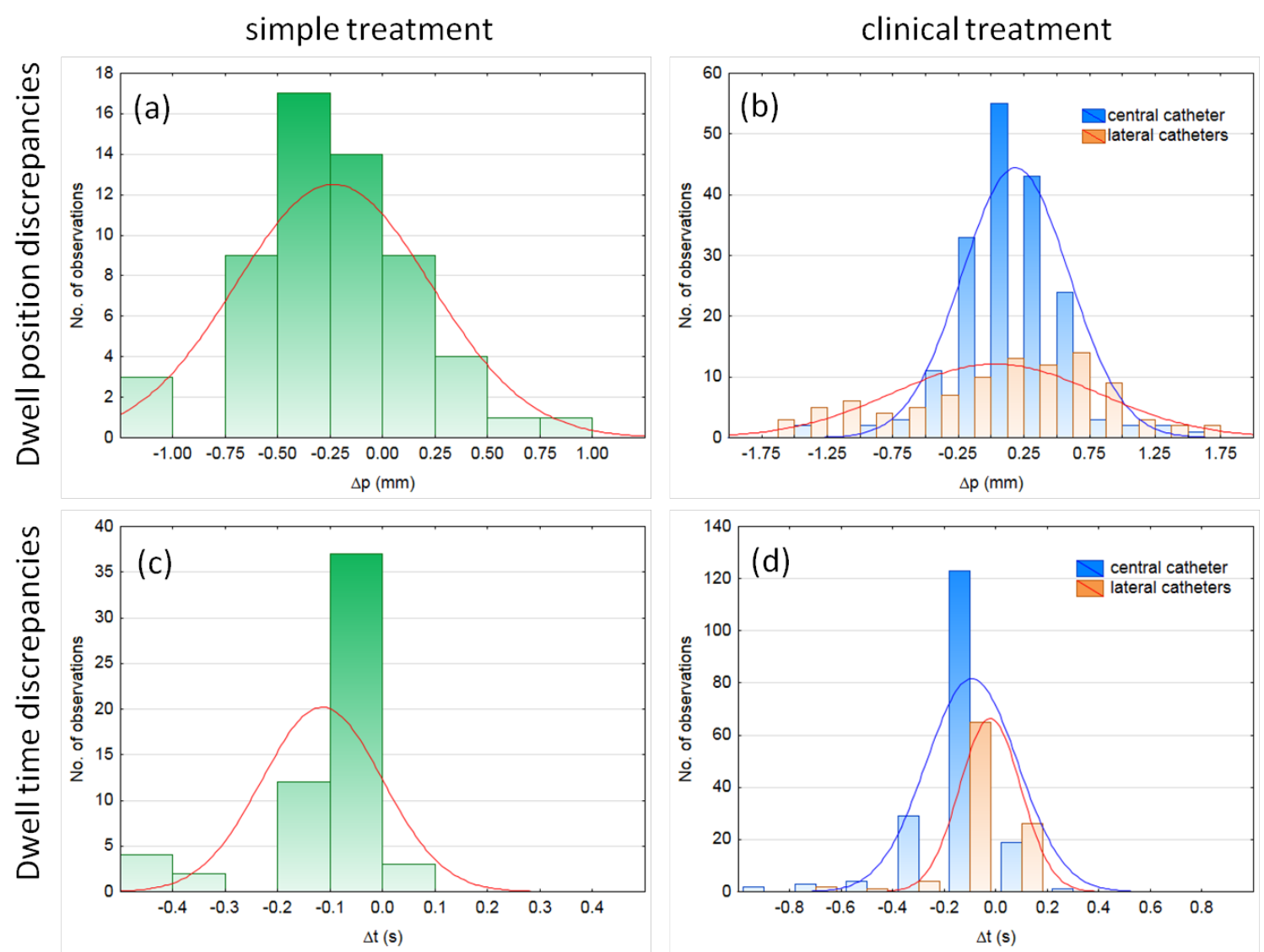

Figure 5 


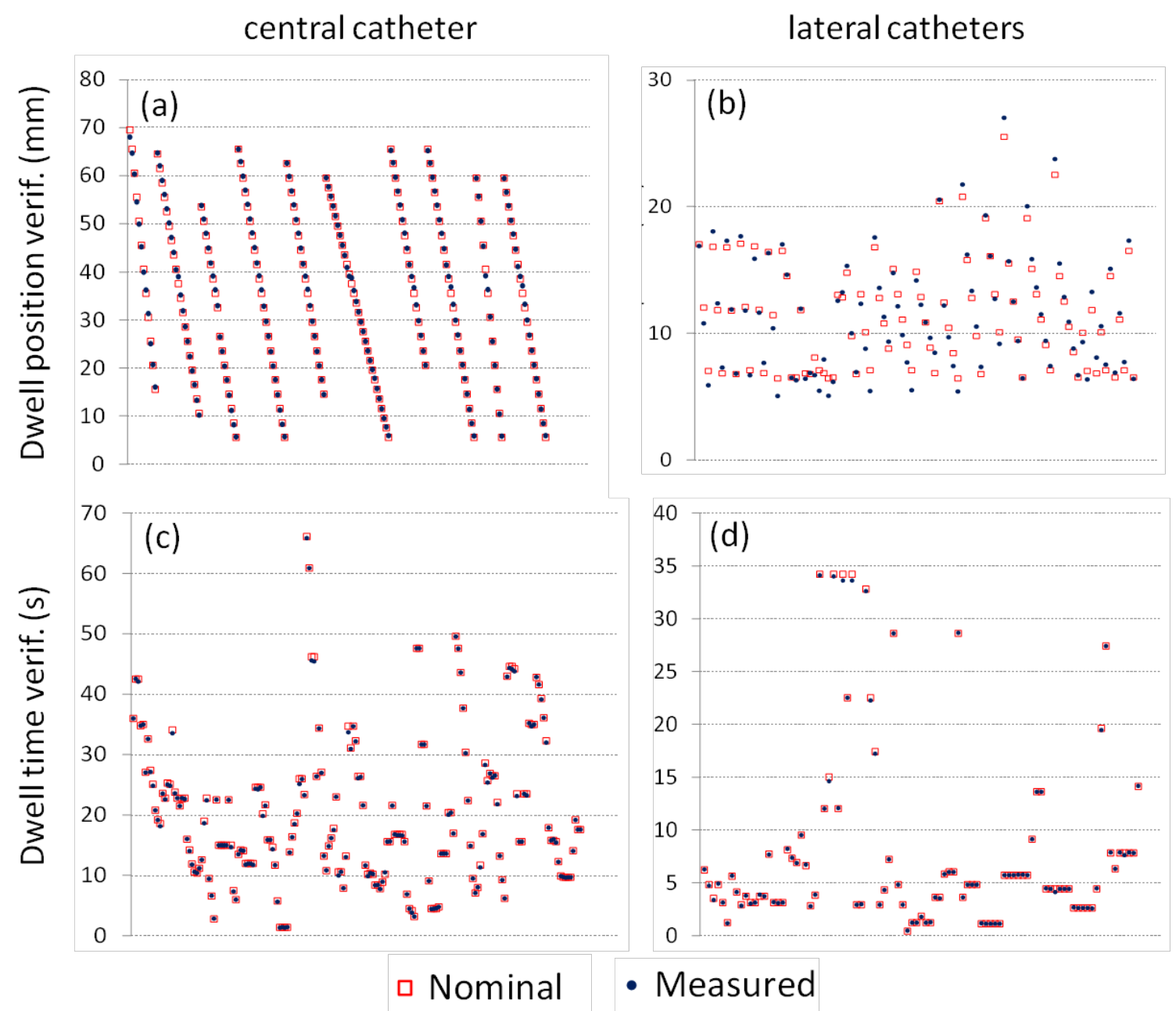

Figure 6 